\title{
Zur Fehlerbreite der manometrischen Blutgasanalyse nach van Slyke
}

\author{
Von \\ C. Albers, F. Kappey und P. Thiele \\ Aus dem W. G. Kerckhoff-Institut der Max-Planck-Gesellschaft, Bad Naubeim (Direktor: Prof. Dr. R. Thauer)
}

(Der Schriftleitung zugegangen am 1. Juli 1963)

\begin{abstract}
Auf Grund von 278 manometrischen van Slyke-Analysen werden die Fehlermöglichkeiten bei der Bestimmung der $\mathrm{O}_{2^{-}}$und $\mathrm{CO}_{2}$-Konzentration im Blut bei Routineuntersuchungen besprochen. Mit Hilfe statistischer Verfahren und der Gaußschen Fehlerrechnung werden zufällige und systematische Fehler getrennt und der Einflu $\beta_{\text {der }}$ einzelnen Teilschritte der Analyse auf den Gesamtfehler abgeschätzt. Der mittlere Fehler der einfachen $\mathrm{CO}_{2}$-Analyse an einem Apparat betrug 0,16 Vol.\%, der der einfachen $\mathrm{O}_{2}$-Analyse $0,07 \mathrm{Vol} \%$. Bei der kombinierten $\mathrm{CO}_{2}-$ und $\mathrm{O}_{2}$-Bestimmung waren die Fehler etwa doppelt so hoch. Bei gleichzeitigen Analysen an 4 Apparaten lag der Fehler nach Korrektur der systematischen Abweichungen für $\mathrm{CO}_{2}$ bei $0,46 \mathrm{Vol}$. \% und für $\mathrm{O}_{2}$ bei $0,19 \mathrm{Vol}$.\%.
\end{abstract}

\begin{abstract}
The possible error in the routine determination of blood $\mathrm{O}_{2}$ and $\mathrm{CO}_{2}$ is discussed on the basis of 278 manometric van Slyke analyses. The random and systematic errors are separated by statistical methods and the Gaussian error calculation and the contribution to the total error of each step of the analysis is estimated. The standatd deviation for the simple $\mathrm{CO}_{2}$ analysis in one apparatus was 0.16 vol.\%, for $\mathrm{O}_{2}$ analysis $0.07 \mathrm{vol} . \%$. In the combined $\mathrm{CO}_{2}$ and $\mathrm{O}_{2}$ determination the errors were approx. twice as high. Simultaneous analyses in 4 apparatuses gave, after correction for systematic deviations, an error of 0.46 vol. $\%$ for $\mathrm{CO}_{2}$ and 0.19 vol. $\%$ for $\mathrm{O}_{2}$.
\end{abstract}

Seit der Einführung des Herzkatheters haben die blutgasanalytischen Methoden weit verbreitetes Interesse gefunden, liefern sie doch die Daten, mit denen klinisch-diagnostisch wichtige Größen wie z. B. das Herzzeitvolumen, Shuntvolumina, Diffusionskapazität der Lunge usw. berechnet werden können. Will man aus solchen abgeleiteten Größen weiterreichende Schlüsse ziehen, muß man sich aber über die Fehlergrenzen dieser Größen Klarheit verschaffen, z. B. durch Anwendung der Gaußschen Fehlerrechnung, die ihrerseits die Kenntnis der Fehlerbreite der angewandten Blutgasanalysen voraussetzt. - Über die Fehlerbreite der van Slyke-Analysen liegen mehrere Angaben in der Literatur vor, die aber alle von besonders geübten Untersuchern stammen und deshalb eine Genauigkeit wiedergeben, die im Routinebetrieb selten erreicht werden dürfte, zumal wenn die Blutgasanalysen von mehreren Untersuchern an mehreren Apparaten durchgeführt werden. Im folgenden soll deshalb über die Ergebnisse einer speziell der Ermittlung der Fehlergrenzen im Routinebetrieb gewidmeten Versuchsreihe berichtet und gezeigt werden, welchen Punkten bei der Analyse bzw. bei der Herstellung der Apparate besondere Aufmerksamkeit zu schenken ist.

\section{Methoden}

Die Durchführung der einfachen und kombinierten $\mathrm{CO}_{2}$ - und $\mathrm{O}_{2}$-Analysen folgte den Vorschriften von vaN SLYKE (1). Die saure Kaliumferricyanidlösung wurde ohne Harnstoff verwendet. Die Kammern der van Slyke-Apparate und die Oswald-Pipetten wurden durch Auswiegen mit Quecksilber geeicht, das zur Einstellung der Menisken mit Wasser überschichtet war (2). Bei jeweils 10 maligem Auswiegen ergab sich mit Hilfe einer einfachen Varianzanalyse als mittlere Streuung ,innerhalb einer Meßreihec $\pm 0,00204$, so daß die Kalibrierung der van Slyke-Kammer auf $0,00204 / \sqrt{10}= \pm 0,00065 \mathrm{ml}$ genau war. Die Genauigkeit der Kalibrierung der Oswald-Pipetten bei Úberschichtung des Quecksilbers mit Wasser lag in der gleichen Größenordnung $( \pm 0,0007 \mathrm{ml}$ ), bei Überschichten mit Blut jedoch etwas höher. Die Daten der verwendeten Apparate und Pipetten sind in Tabelle 1 zusammengestellt. - Die Analyșen wurden an 4 Apparaten gleichzeitig von 4 durchschnittlich geübten Untersuchern in Doppelbestimmungen ausgeführt und ohne Auslese für die Auswertung verwendet, $d$. h. es wurden auch offensichtliche "Ausreißer" mit berücksichtigt, die man normalerweise durch Ausführung einer dritten Analyse zu eliminieren versucht hätte. Die exhaltenen Feblerangaben dürften deshalb eher zu hoch sein. Die statistische Auswertung erfolgte zumeist mit Hilfe einer mehrfachen Varianzanalyse. Die Ergebnisse werden in der Reihenfolge der zu beachtenden Fehlerquellen besprochen.

Tab. 1

Kalibrierung der verwendeten van Slyke-Kammern und OswaldPipetten

\begin{tabular}{ccc}
\hline Apparat Nr. & $\begin{array}{c}\text { Volumen bei } \\
\text { Marke 2,000 }\end{array}$ & $\begin{array}{c}\text { Volumen der } \\
\text { Oswald-Pipette }\end{array}$ \\
\hline I & 1,993 & 1,000 \\
II & 2,012 & 1,002 \\
III & 1,982 & 1,007 \\
IV & 2,004 & 1,000 \\
\hline
\end{tabular}

1. Die Ablesegenauigkeit wird unter optimalen Bedingungen (Sauberkeit der Kammer, Durchsichtigkeit der Medien usw.) mit 0,1 mm anzusetzen sein. Für die Differenz aus 2 Druckablesungen erhält man nach Gauß einen mittleren Fehler von $0,1 \sqrt{2}= \pm 0,14 \mathrm{~mm}$.

2. Der einfacbe Hantierungsfebler, d. h. der durch das Abmessen der Reagenzien, die Evakuierung und Einstellung der Menisken bewirkte Fehler läßt sich durch 
Bestimmung der Leerwerte (c-Korrekturen) bestimmen. Bei zusammen 118 Leerwert-Bestimmungen an 4 Apparaten lieferte die Varianzanalyse als Streuung ,innerhalb einer Meßreihe an einem Apparat" $\pm 0,21 \mathrm{~mm}$, also einen nur wenig über den Ablesefehler herausgehenden Wert.

3. Der totale Hantierungsfebler entspricht der Reproduzierbarkeit der van Slyke-Analysen an einem Apparat und wird durch die mittlere Abweichung bei Doppelanalysen erfaßt. Er ist die Summe aus dem einfachen Hantierungsfehler zuzüglich des Pipettierfehlers, des Fehlers durch unvollständige Extraktion der Blutgase und des Fehlers der bei der Absorption der einzelnen Gasfraktionen auftritt. Er ist, wie Tabelle 2 zeigt, von der Art der Blutgasanalyse abhängig, und betrug bei früheren und den jetzigen Untersuchungen

für die einfache $\mathrm{CO}_{2}$-Bestimmung $\pm 0,16 \mathrm{Vol} . \%$, für die einfache $\mathrm{O}_{2}$-Bestimmung $\pm 0,07 \mathrm{Vol} \%$ und für die kombinierte $\mathrm{CO}_{2}$ - und $\mathrm{O}_{2}$-Analyse $\pm 0,31 \mathrm{Vol}$. \% bei $\mathrm{CO}_{2}\left( \pm 0,12 \mathrm{Vol}\right.$. $\%$ bei $\left.\mathrm{O}_{2}\right)$. Dieser Fehler ergab sich bei der Analyse von 19 Blutproben mit $4 \times 2$ Doppelanalysen an 4 Apparaten als „Binnenfachstreuung" der doppelten Varianzanalyse mit 2 Werten je Fach. (Die Streuung, ,innerhalb einer Meßreihe an einem Apparat" ist uninteressant, da sie nur ein $\mathrm{Maß}$ für die Breite des Untersuchungsbereiches ist, der für $\mathrm{CO}_{2} 15-86 \mathrm{Vol}$ \% und für $\mathrm{O}_{2} 8-24 \mathrm{Vol}$ \% $\%$ betrug. Die Streuung „zwischen den Meßreihen“ wird bei der Besprechung des systematischen Fehlers diskutiert.)

Beim Pipettierfehler ist ein systematischer (s. Tabelle 3) und ein zufälliger, durch das ungleichmäßige Auslaufen der Oswald-Pipette bedingter Anteil zu unterscheiden. Um letzteren zu ermitteln, wurde eine Oswald-Pipette je $10 \mathrm{mal}$ mit Quecksilber ausgewogen, wobei das

\section{Tab. 2}

Fehlerquellen und Reproduzierbarkeit der van Slyke-Analyse. Berechnung des mittleren Fehlers in Vol.\% mittels Varianzanalyse, Umrechnung in mm mittlerer Druckdifferenz unter Annahme einer Analysentemperatur von $20^{\circ}$ für Blutproben von $1 \mathrm{ml}$, Flüssigkeitsvolumen $3,5 \mathrm{~m} l$ und eingestelltes Gasvolumen von $2,000 \mathrm{ml}$. $\mathrm{n}=$ Zahl der zugrunde liegenden Analysen

\begin{tabular}{|c|c|c|c|}
\hline Art des Fehlers & $\mathbf{n}$ & $\begin{array}{c}\text { mittl. Fehler } \\
\text { Vol. } \%\end{array}$ & $\mathrm{~mm}$ \\
\hline $\begin{array}{l}\text { Ablesefehler } \\
\text { einfacher Hantierungsfehler } \\
\quad \text { (Leerwert) }\end{array}$ & - & - & $\begin{array}{l}0,14 \\
0,21\end{array}$ \\
\hline $\begin{array}{l}\text { totaler Hantierungsfehler (1 App.) } \\
\text { einfache } \mathrm{CO}_{2} \text {-Analyse } \\
\text { einfache } \mathrm{O}_{2} \text {-Analyse nach } \\
\text { AlBERS und Mitarbeitern (4) } \\
\text { kombinierte Analyse } \\
\mathrm{CO}_{2} \\
\mathrm{O}_{2}\end{array}$ & $\begin{array}{r}24 \\
102 \\
102\end{array}$ & $\begin{array}{l}0,07 \\
0,31 \\
0,12\end{array}$ & $\begin{array}{l}1,16 \\
0,49\end{array}$ \\
\hline $\begin{array}{l}\text { totaler Fehler bei Analysen an } \\
4 \text { App. } \\
\mathrm{CO}_{2} \\
\mathrm{O}_{2}\end{array}$ & $\begin{array}{l}136 \\
152\end{array}$ & $\begin{array}{l}0,46 \\
0,19\end{array}$ & $\begin{array}{l}1,73 \\
0,77\end{array}$ \\
\hline
\end{tabular}

Tab. 3

Einfluß des Haematokrits auf das Auslaufvolumen einer OswaldPipette bei Auswiegen mit Quecksilber und Reduktion auf $20^{\circ}$. (Mittelwerte aus je 10 Messungen.)

\begin{tabular}{lcccc}
\hline Haematokrit (\%) & 0 & 32 & 51 & 64 \\
$\begin{array}{l}\text { Volumen (ml) } \\
\begin{array}{l}\text { Standardab- } \\
\text { weichung }\end{array}\end{array}$ & 1,0184 & 1,0155 & 1,0131 & 1,0142 \\
& $\pm 0,0007$ & $\pm 0,0008$ & $\pm 0,0016$ & $\pm 0,0016$ \\
\hline
\end{tabular}

Quecksilber mit Wasser oder mit Blut mit steigendem Haematokrit überschichtet wurde. Die Ergebnisse zeigt Tabelle 3. Man erkennt deutlich, daß sowohl ein systematischer Fehler vorliegt (das aus der Pipette auslaufende Volumen ist um so kleiner, je höher der Haematokrit ist), als auch, daß die Pipette bei höherem Haematokrit ungleichmäßiger ausläuft (Anstieg der Standardabweichung). Die systematische Abweichung zwischen Wasser und Blut war in dieser Reihe mit $0,005 \mathrm{~m} l$ etwas kleiner als die von Severinghaus und Mitarbb. (3) gefundene von $0,008 \mathrm{~m} /$. Der Zufallsfehler betrug für Blut von mittleren Hämoglobingehalt rund $0,16 \%$. Berechnet man nach Gauß den Einfluß dieses Fehlers und des einfachen Hantierungsfehlers auf das Analysenergebnis, so erhält man für eine $\mathrm{CO}_{2}$ Konzentration von $40 \mathrm{Vol} \%$ einen Fehler von $\pm 0,09 \mathrm{Vol} . \%$ und für eine $\mathrm{O}_{2}$-Konzentration von 20 Vol. \% einen Fehler von $\pm 0,06$ Vol. \%. Der für die einfache $\mathrm{O}_{2}$-Bestimmung gefundene totale Hantierungsfehler entspricht dieser Größenordnung, der für die einfache $\mathrm{CO}_{2}$-Bestimmung gefundene liegt etwa doppelt so hoch und die bei der kombinierten Methode erhaltenen vor allem für $\mathrm{CO}_{2}$ wesentlich höher. Hierfür sind 2 Gründe maßgeblich:

a) Beim Einstellen des Flüssigkeitsmeniskus auf die Marke $2,000 \mathrm{ml}$ befindet sich ein dünner Flüssigkeitsfilm an der Kammerwand, so $\mathrm{da}$ das wahre Gasvolumen etwas kleiner als bei der Kalibrierung mit Wasser sein wird. Dieser Film macht sich vor allem bei der Verwendung des sauren Kaliumferricyanid infolge der Ausfällungen von saurem Hämiglobin bemerkbar, die erst nach Zugabe der $\mathrm{NaOH}$ wieder großenteils in Lösung gehen. Der Einfluß dieses Fehlers ist beim $\mathrm{CO}_{2}$ deshalb wesentlich größer als beim $\mathrm{O}_{2}$, dort aber auch noch vorhanden.

b) Die Extraktion der Gase kann unvollständig sein. Hinzu kommt beim $\mathrm{CO}_{2}$ die Gefahr der Rückabsorption während der ersten Druckablesung, die sich vor allem bei hohen $\mathrm{CO}_{2}$-Werten in einem unaufhörlichen Druckabfall sehr störend bemerkbar machen kann. Während sich für den zuletzt genannten Fehler keine Abhilfe schaffen läßt, hat sich bei unseren Versuchen gegen die unvollständige Extraktion statt der mehrfachen Extraktion bis zur Druckkonstanz von $\mathrm{p}_{1}$ folgendes Verfahren bewährt: Man exhöht die Milchsäurekonzentration der Stammlösung auf $0,2 n$, so da $\beta$ das Reagenz einer 0,1 $n$ Lösung entspricht, und läßt während der letzten Minute der Extraktion den $\mathrm{Hg}$-Spiegel vom unteren geraden Rohrteil mit der Marke $50 \mathrm{~m} / 4-5 \mathrm{mal}$ langsam bis gerade eben in die birnenförmige Erweiterung der Kammer steigen. Dies ist unumgänglich, wenn sich die Marke $50 \mathrm{~m} / \mathrm{sehr}$ weit unten im geraden Rohrteil befindet, was von den Herstellern vermieden werden sollte.

\section{Die systematischen Febler}

Während sich die Reproduzierbarkeit der Analysen an einem Apparat durch sorgfältiges Arbeiten auf die Größenordnung von 0,1 Vol.\% bringen läßt, zeigt der 
Analysenvergleich an mehreren Apparaten ein ungünstigeres Bild, da nun noch systematische Abweichungen zwischen den Apparaten zu berücksichtigen sind. Diese ließen sich bei der Serie von 19 Doppelanalysen an je 4 Apparaten schon daran erkennen, daß die Ergebnisse an den einzelnen Apparaten regelmäßig in der Richtung voneinander differierten. Bezogen auf den Apparat Nr. 1 der Tabelle 1 fand sich für $\mathrm{CO}_{2}$ am 2. Apparat $18 \mathrm{mal}$, am 3. und 4. Apparat je $17 \mathrm{mal}$ ein niedrigerer Wert; für $\mathrm{O}_{2}$ betrug die $\mathrm{Zahl}$ der negativen Differenzen am 2. und 4. Apparat 17 sowie am 3. Apparat 15. Schon mit dem einfachen Vorzeichentest erweisen sich diese Abweichungen als überzufällig. Die genauere statistische Analyse zeigte, daß es sich nicht um einen additiven Fehler handelt, sondern daß sich der Fehler durch einen Faktor korrigieren läßt. Wenn man Apparat 1 als Bezugsapparat wählte, ergab sich der Korrekturfaktor mit Hilfe der Regressionsberechnung, wobei der jeweilige Wert von Apparat 1 als unabhängige, der entsprechende Wert des anderen Apparates als abhängige Variable behandelt wurde. Die Regression wurde für die durch den Nullpunkt gehende Gerade von der Form $y=b x$ berechnet. Die erhaltenen Koeffizienten verzeichnet Tabelle 4. An diesen Korrekturfaktoren ist zweierlei bemerkenswert: 1. Der Korrekturfaktor ist für $\mathrm{CO}_{2}$ stets höher als $\mathrm{O}_{2}$. 2. Berechnet man den nach der Kalibrierung der Kammer zu erwartenden Faktor, so liegt dieser stets niedriger als die für $\mathrm{CO}_{2}$ und $\mathrm{O}_{2}$ gefundenen. Diese Differenzen sind statistisch signifikant. Ihre Ursache liegt in der erwähnten Bildung eines Flüssigkeitsfilms, die das wahre Gasvolumen verfälscht. $\mathrm{Da}$ die Filmbildung vom Rohrdurchmesser, von der Benetzbarkeit der Wand (und damit von der Reinigung der Kammer nach der Analyse!) und von der Form der oberen Aufblasung der Kammer abhängt, wäre es wünschenswert, die Abmessungen der Kammer einheitlich zu normieren.

Von größter Wichtigkeit ist die Feststellung, daß der durch Kalibrieren von Pipette und Kammer errecbnete Korrekturfaktor die systematische Abweicbung nicht genau vorherzusagen gestattet, so daß man bezüglich der

Tab. 4

Systematische Differenzen zwischen van Slyke-Apparat I-IV. $\mathrm{M}(\rightarrow)$ und $\mathrm{M}(+)=\mathrm{Zahl}$ der negativen bzw. positiven Differenzen zu Apparat $\mathrm{I} ; \mathrm{b}_{\mathrm{CO}_{2}}$ und $\mathrm{b}_{\mathrm{O}_{2}}=$ Regressionskoeffizient der jeweiligen Regressionsgleichung; Gaskonzentration App. II (III, IV) $=\mathrm{b}$ Gaskonzentration App. I; $b_{\mathrm{Kal}}=$ nach der Kalibrierung zu erwartender Wert für $b$.

\begin{tabular}{lccc}
\hline \multicolumn{1}{c}{ App. Nr. } & II & III & IV \\
$\mathrm{CO}_{2} \mathrm{M}(-)$ & 18 & 13 & 17 \\
$\mathrm{M}(+)$ & 0 & 4 & 1 \\
$\mathrm{P}$ (Vorzeichentest) & $<1 \%$ & $<2 \%$ & $<1 \%$ \\
\hline $\mathrm{O}_{2} \mathrm{M}(-)$ & 17 & 15 & 17 \\
$\mathrm{M}(+)$ & 2 & 4 & 0 \\
$\mathrm{P}($ Vorzeichentest $)$ & $<1 \%$ & $<1 \%$ & $<1 \%$ \\
\hline $\mathrm{b}_{\mathrm{CO}_{2}}$ & 1,0351 & 1,0066 & 1,0237 \\
$\mathrm{~b}_{\mathrm{O}_{2}}$ & 1,0333 & 1,0044 & 1,0208 \\
$\mathrm{~b}_{\text {Kal }}$ & 1,0095 & 0,9945 & 1,0055 \\
\hline
\end{tabular}

Tạb. 5

Mittelwerte von Doppclanalysen an 4 van Slyke-Apparaten nach Korrektur der systematischen Abweichung.

$\mathrm{CO}_{2}$-Werte in Vol.\% $\quad \mathrm{O}_{2}$-Werte in Vol. $\%$

\begin{tabular}{|c|c|c|c|c|c|c|c|c|}
\hline App.-I & 1 & II & III & IV & I & II & III & IV \\
\hline & & & & & 17,64 & 17,14 & 17,37 & 17,62 \\
\hline & 48,44 & 46,58 & 48,05 & 47,40 & 18,87 & 18,73 & 18,63 & 18,90 \\
\hline & 35,54 & 34,86 & 35,33 & 35,07 & 19,60 & 19,63 & 20,02 & 19,78 \\
\hline & 40,57 & 40,98 & 40,90 & 39,09 & 21,89 & 21,88 & 22,04 & 21,63 \\
\hline & 41,23 & 41,22 & 41,47 & 39,93 & 21,41 & 21,36 & 21,35 & 21,21 \\
\hline & 44,85 & 44,83 & 45,09 & 44,69 & 16,20 & 16,00 & 16,11 & 16,13 \\
\hline & 47,99 & 48,55 & 48,21 & 48,61 & 10,96 & 10,76 & 10,96 & 11,06 \\
\hline & 54,42 & 54,49 & 53,94 & 54,65 & 12,19 & 12,65 & 12,56 & 11,49 \\
\hline & 47,52 & 48,05 & 47,84 & 48,39 & 17,11 & 17,22 & 17,11 & 17,24 \\
\hline & 40,89 & 40,99 & 41,16 & 41,50 & 13,64 & 13,59 & 13,83 & 13,92 \\
\hline & 44,07 & 43,75 & 43,93 & 44,56 & 18,68 & 19,03 & 18,73 & 19,13 \\
\hline & 27,89 & 27,72 & 28,25 & 28,02 & 16,49 & 16,65 & 16,49 & 16,42 \\
\hline & 38,44 & 38,56 & 37,62 & 37,10 & 22,83 & 22,75 & 22,72 & 22,71 \\
\hline & 38,51 & 39,24 & 39,45 & 38,38 & 24,20 & 24,43 & 24,15 & 24,28 \\
\hline & 37,20 & 37,79 & 37,99 & 38,13 & 12,61 & 13,07 & 13,04 & 12,84 \\
\hline & 19,26 & 19,01 & 19,09 & 19,36 & 11,32 & 10,90 & 11,16 & 11,26 \\
\hline & 27,25 & 27,13 & 27,20 & 27,51 & 13,62 & 13,41 & 13,20 & 13,43 \\
\hline & 15,67 & 15,60 & 15,39 & 15,78 & 8,03 & 7,94 & 7,97 & 7,87 \\
\hline & 86,61 & 86,66 & 85,99 & 87,18 & 11,90 & 11,83 & 11,74 & 11,79 \\
\hline & 40.91 & 40.89 & 40.94 & 40.85 & 16.27 & 16.26 & 16.27 & 16.25 \\
\hline
\end{tabular}

Absolutwerte der Gaskonzentrationen mit einem Fehler von schätzungsweise $1 \%$ zu rechnen hat. Die Bestimmung des $\mathrm{CO}_{2}$-Gehalts bekannter Bicarbonatlösungen ist nur ein sehr bedingtes Hilfsmittel, da bei wässerigen L̇ösungen der Flüssigkeitsfilm viel weniger ins Gewicht fällt als bei Plasma- oder Blutanalysen. Tabelle 5 zeigt die mit dem Regressionskoeffizienten der $\mathrm{Ta}$ belle 4 korrigierten Mittelwerte der Doppelanalysen an den Apparaten I-IV. Eine systematische Differenz ist, wie aus der Übereinstimmung der Mittelwerte und aus dem negativen Ausfall des Vorzeichentestes hervorgeht, nicht mehr zu erkennen. Die gute Übereinstimmung der Mittelwerte zeigt jedoch nur das Fehlen einer systematischen Abweichung an und darf nicht, wie es oft fälschlich geschieht, auch zur Beurteilung des zufälligen Fehlers herangezogen werden. Dieser läßt sich aus den Daten der Tabelle 5 mit Hilfe der Varianzanalyse ermitteln, wobei als Streuungsmaß die Varianz ,innerhalb der Zeilen" gewertet werden kann, da es sich zeigte, $\mathrm{da} \beta$ die Varianz "zwischen den Spalten" und die Wechselwirkung "Spalte-Zeile“ als gleich anzusehen waren. Es ergab sich so ein mittlerer Fehler für die $\mathrm{CO}_{2}$-Analyse von $0,46 \mathrm{Vol} . \%$ und für die $\mathrm{O}_{2}$-Analyse von 0,19 Vol.\% (Tabelle 2). Diese sowohl durch apparative Fehlerursachen wie natürlich auch durch den Trainingsgrad der Untersucher bedingten Fehler lassen sich durch Intensivierung und Verlängerung der Einarbeitungszeit sicher verkleinern. Immerhin sollte man im alltäglichen Routinebetrieb vorsichtshalber die angegebene Fehlerbreite in Rechnung stellen.

Welche praktischen Schlußfolgerungen ergeben sich aus diesen Messungen? Hier ist zu unterscheiden zwischen Messungen, bei denen es auf die absoluten Gaskonzentrationen ankommt (z. B. bei der Bestimmung des 
$\mathrm{CO}_{2}$-Druckes aus $\mathrm{pH}$ und $\mathrm{CO}_{2}$-Gehalt, bei der gasometrischen Bestimmung des Hämoglobingehalts mit der $\mathrm{CO}$ - oder $\mathrm{O}_{2}$-Kapazität), oder ob Relativbeziebungen von Blutgaswerten interessieren, wie z. B. bei der Bestimmung. der $\mathrm{O}_{2}$-Sättigung aus $\mathrm{O}_{2}$-Gehalt der aktuellen Blutprobe und $\mathrm{O}_{2}$-Kapazität, bei der $\mathrm{Be}-$ stimmung der arteriovenösen $\mathrm{O}_{2}$ und $\mathrm{CO}_{2}$-Differenzen oder bei der Bestimmung des $\mathrm{CO}_{2}$-Druckes mit der Tonometer-Methode aus dem $\mathrm{CO}_{2}$-Gehalt der aktuellen und dem der tonometrierten Blutprobe. Im ersten Fall muß man den vollen Fehler einschließlich der durch die systematischen Abweichungen bedingten in Rechnung stellen, jedoch können die An̈alysen einer Serie von mehreren Untersuchern an mehreren van Slyke-Ápparaten durchgeführt werden. Im zweiten Fall ist es dagegen vorteilhaft, die zusammengehörigen Blutgaswerte an einem Apparat bestimmen zu lassen, da sich die systematischen Fehler dann nur wenig auswirken. Unzweckmäßig wäre dagegen eine Aufteilung der Analysen auf mehrere Apparate derart, daß z. B. alle Tonometerwerte an einem, alle arteriellen Blutproben an einem zweiten und alle venösen Werte an einem dritten Apparat bestimmt werden. Bei solchem Vorgehen wären die abgeleiteten Größen wie prozentuale Sättigung, $\mathrm{CO}_{2}$-Druck usw. ebenfalls systematisch verfälscht und nicht nur mit dem Zufallsfehler behaftet.

\section{Literatur}

1. Peters, J. D. und D. D. van Slyke, Quantitative Clinical Chemistry, II., Methods, London (1932). - 2. OpITz, E. und H. BArtels, Gasanalyse, in Hoppe-Seyler-Thierfelder's Handbuch der Physiologisch und Pathologisch-Chemischen Analyse. 10. Aufl. Bd. II., S. 183. Springer-Verlag, Berlin-Göttingen-Heidelberg (1955). - 3. Severinghaus, J. W., M. Stuppel und A. F.
Brandley; J. appl. Physiol. 9, 189 (1956). - 4. Albers, C., W. Brendel und W. Usinger, Pflügers Arch. ges. Physiol. Menschen Tiere 262, 256 (1956). - 5. Bartels, H., E. BücherL, C. W. Hertz, G. Rodewlad und M. SCHWab, Lungenfunktionsprüfungen. Springer-Verlag, Berlin-Göttingen-Heidelberg (1959). 6. SChWAB, M. und H. Wisser, Klin. Wochschr. 36, 741 (1958).

\section{Discussion of T.K. WiTH's paper on acute intermittent porphyria}

with some comments on the problem of classification ${ }^{1}$ )

C. J. WATSON

From the Department of Medicine, University of Minnesota Hospital, Minneapolis, Minn., USA

(Der Schriftleitung zugegangen am 26. November 1963)

A recent issue of this Journal (October 1963) presented a study by TORBEN WITH (1) entitled "Acute intermittent porphyria. Family studies on the excretion of PBG and delta-ALA with ion exchange chromatography". In this Dr. WITH speaks of two concepts of hepatic porphyria, one which he terms "unitary", the other "molecular". According to the former all of the clinical variants of hepatic porphyria have the same

1) Aided by grants from the U.S. P. H. S. and the John and Mary Briggs Porphyria Research Fund. basic (genetic) abnormality, while the latter refers to differing genetic disturbances for each of the principal variants. In discussing the "unitary" concept he has referred to my Chester Jones Lecture of 1960 (2) stating "according to the unitary hypothesis based on Warson's study of 268 cases from USA, the varying manifestations of hepatic porphyria can be explained by one gene and modifications due to environmental factors". This would suggest that I have disregarded the eviderice which DeAN and BARNes (3), WALDENSTRÖM (4) 